\title{
Mandibular Tori as Bone Grafts: An Alternative Treatment for Periodontal Osseous Defects - Clinical, Radiographic and Histologic Morphology Evaluation
}

\author{
${ }^{1}$ Khalid S Hassan, ${ }^{2}$ Adel Al-Agal, ${ }^{3}$ Adel I Abdel-Hady, ${ }^{4}$ Wael M Swelam, ${ }^{5}$ Reda F Elgazzar
}

\begin{abstract}
Objectives: The present study was designed to evaluate the clinical, radiographic and histochemical significance of using the mandibular tori as autogenous bone graft for treatment of intraosseous defects in patients with chronic periodontitis.

Materials and methods: Twenty-eight sites from 14 patients with chronic periodontitis were included in this study. Each patient was treated with split mouth design; one site received torus mandibularis bone graft and the other site received a full-thickness flap alone. Histopathologic assessment was evaluated on removal of torus mandibularis to evaluate its histologic structure and by the end of the study 9 month later. Clinical and radiographic parameters were re-evaluated at 3 months interval for 1 year.
\end{abstract}

Results: The results of the present study revealed significant gain in the clinical attachment level (CAL) $(88.4 \%, 4.53 \pm$ $0.06 \mathrm{~mm}$ ) for torus mandibularis sites compared to $(39.7 \%$, $2.01 \pm 0.04 \mathrm{~mm}$ ) for full-thickness flap. Moreover, there was a reduction in the probing pocket depth (PPD) of $(75.4 \%$, $5.75 \pm 0.12 \mathrm{~mm})$ for torus mandibularis sites and $(49.6 \%, 3.73$ $\pm 0.14 \mathrm{~mm}$ ) for sites treated with a full-thickness flap only; CAL and PPD differences were significant at $p$-value $\leq 0.01$. Concomitantly, significant radiographic increase in the bone height and density were recorded in the test group.

Conclusion: The use of mandibular tori as autogenous bone graft could provide benefits as a periodontal therapeutic

\footnotetext{
${ }^{1}$ Department of Preventive Dental Sciences, Division of Periodontics, College of Dentistry, University of Dammam Dammam, Saudi Arabia; Department of Oral Medicine and Periodontology College of Dentistry, Al-Azhar University, Egypt

${ }^{2}$ Department of Preventive Dental Sciences, Division of Periodontics, College of Dentistry, University of Dammam Dammam, Saudi Arabia

${ }^{3}$ Department of Oral and Maxillofacial Surgery, College of Dentistry, University of Dammam, Dammam, Saudi Arabia

${ }^{4}$ Department of Oral and Maxillofacial Pathology, College of Dentistry, Tiba University, Saudi Arabia

${ }^{5}$ Department of Oral and Maxillofacial Surgery, Manitoba University, Canada; Department of Oral and Maxillofacial Surgery, Tanta University, Egypt

Corresponding Author: Khalid S Hassan, Associate Professor, Department of Preventive Dental Sciences, Division of Periodontics, College of Dentistry, University of Dammam Dammam 31441, PO Box 1982, Saudi Arabia, Phone: +966 54-476-0872, e-mail: hassan.khalid56@yahoo.com
}

modality and enhance regenerative potential of periodontal intraosseous defects.

Keywords: Autogenous bone grafts, Chronic periodontitis, Mandibular tori.

How to cite this article: Hassan KS, Al-Agal A, Abdel-Hady AI, Swelam WM, Elgazzar RF. Mandibular Tori as Bone Grafts: An Alternative Treatment for Periodontal Osseous DefectsClinical, Radiographic and Histologic Morphology Evaluation. J Contemp Dent Pract 2015;16(3):192-200.

\section{Source of support: Nil}

Conflict of interest: None

\section{INTRODUCTION}

The optimal goal of periodontal therapy is to resolve inflammation and control disease progression where successful regeneration of a new attachment apparatus replaces the destroyed one. ${ }^{1}$ Unfortunately, this has long been an elusive goal for more than a century. ${ }^{2-4}$ Regeneration is defined as the reproduction or reconstitution of the lost structures of the periodontium restoring its architecture and function. Histological evidence of periodontal regeneration must include new alveolar bone, reestablishment of periodontal ligament and cementum. ${ }^{5,6}$ Bone grafts and synthetic substitutes have been utilized to achieve these therapeutic goals.

Different treatment modalities have been attempted to restore the lost periodontal apparatus with varying outcomes. ${ }^{7-9}$ Extaoral or intraoral autogenous bone grafts showed the best regenerative results histologically. ${ }^{10-12}$ Demineralized freeze-dried bone showed similar results. ${ }^{13,14}$ Consequently, autogenous bone grafts have been widely used for regenerative procedures. A variety of intraoral sites have been utilized for harvesting, including extraction sockets and bony exostoses. Several studies have reported on the clinically successful use of autogenous bone grafts harvested from the intraoral sites in the treatment of intrabony defects. ${ }^{15-19}$ These authors show that regenerative procedures including autogenous bone grafting was superior to surgical debridement alone. A resolution of more than $50 \%$ has been shown when using intraorally harvested bone. ${ }^{20,21}$

Bone exotoses, are localized bony protuberances that arise from the cortical plate and sometimes from the 
spongy layer, ${ }^{22}$ with no pathological significance. Torus palatinus and torus mandibulae are two of the most common intraoral exotoses. The other types of exotoses as buccal or palatal exostoses are less commonly encountered. ${ }^{23}$ Torus mandibulae, usually found bilaterally, are localized on the lingual side of the mandible in the premolar region superior to the mylohyoid ridge, first described in 1908 by Fürst, 1908. ${ }^{24,25}$

Mandibular tori may limit tongue space occasionally causing phonetic problems. Therefore, surgical removals of tori are occasionally required for prosthetic reasons. The surface of removed exotoses which are rich with proliferating osteoblasts as well as fibro-cartilaginous capsule that might contain undifferentiated osteoblasts attracted our interest in utilizing it as autogenous bone graft to restore the periodontal bony defects. The present study protocol was designed to evaluate and compare the treatment outcome, clinically, radiographically, and histochemically of the two treatment modalities, fullthickness flap with and without autogenous bone grafting from mandibular tori; in the treatment of intrabony osseous defects.

\section{MATERIALS AND METHODS}

Fourteen patients ( 8 females and 6 males; age range: 37 to 48 years with an average age of $45.3 \pm 3.81$ years) with chronic periodontitis (localized form, less than $30 \%$ involved sites) and having torus mandibularis were selected to participate in this randomized, controlled and parallel clinical design study. Patients were selected from the outpatient clinic, College of Dentistry, University of Dammam, Kingdom of Saudi Arabia. Patients with any systemic illness known to affect the outcomes of periodontal therapy, compromised immune system, pregnancy and/or lactation, smoking or the use of other tobacco products, those taking drugs known to interfere with wound healing, alcohol use habits, allergy or sensitivity to any medication to be used in the study, and those with unacceptable oral hygiene after the reevaluation of phase I therapy were excluded from the study.

The inclusion criteria were the presence of infrabony defect (Vertical bone loss) with loss of CAL of at least $5 \mathrm{~mm}$ and PPD of not less than $6 \mathrm{~mm}$. Twenty-eight sites from fourteen patients were selected using a split mouth design for each patient which was randomly determined through a coin toss. One site (group I) received a fullthickness mucoperiosteal flap and the infrabony defect was filled with Torus mandibularis bone graft. The other site (group II) received a full-thickness flap alone. Each patient was prepared for surgery with an initial phase of therapy which included oral hygiene instructions and scaling and root planing. Approximately 4 weeks after the initial therapy, the patients were reevaluated to assess clinical parameters and plaque control. All patients were required to achieve a good oral hygiene (less than $20 \%$ O'Leary plaque index) done by scaling and root planing and oral hygiene instructions prior to progressing to the surgical phase of therapy.

Full explanation of the research objective was explained to all patients included in this study, and then they were asked to sign a written consent demonstrating their approval of the procedure and publication of its results. In addition, this research was approved by the ethics committee, College of Dentistry, University of Dammam.

The following clinical parameters were assessed at the baseline, 3, 6, 9 and 12 months postoperatively using the same periodontal probe (NUC-15, Hu-Friedy, USA); the gingival index (GI; Lôe and Sillness, 1963), ${ }^{26}$ pocket probing depth (PD) and clinical attachment level (CAL; Ramfjord, 1967). ${ }^{27}$ For 1 year, the clinical and radiographic parameters were re-evaluated at 3 month intervals. The measurements recorded at 6 points (recording the deepest site) around the treated teeth.

\section{Bone Graft Collection}

All procedures were performed under sterile conditions. Prior to surgery, all patients rinsed with $0.12 \%$ chlorhexidine for 30 seconds. A full-thickness mucoperiosteal flap was elevated for torus mandibularis access and for bone graft collection (Fig. 1A). Vertical releasing incisions (one distal or mesial releasing incision with minimal length was elevated to expose the mandibular tori) were performed if necessary for better access. A surgical bur \#701 and 702 (FGOS) in a slow speed handpiece was used under copious irrigation to remove the torus mandibularis. Bone grafts were collected with a bone trap according to the method described by Sivolella et $\mathrm{al}^{28}$ (Omniasurg ASP 100; Omnia srl, Fidenza, PR, Italy). The trap filter was equipped with a removable internal mesh with a pore diameter of $300 \mu \mathrm{m}$. Two distinct systems were used for aspiration and bone collection. One system was used for the control of saliva, bleeding, and was kept at a distance of $1.5 \mathrm{~cm}$ from the osteoctomy site. The other system was sterile and disposable, and comprised a filter for collecting bone chips and a plastic suction tube. The latter was held as close as possible to the osteoctomy site in order to collect the bone debris and reduce the aspiration of saliva (Fig. 1B). The collected tissues were placed in a sterile bone well containing sterile saline solution at room temperature and covered with a cap in order to minimize the risk of contamination. After preparing of the receiving site, the excess of saline solution was removed and only the collected materials were used to fill the intraosseous defects 
in-group I. The flaps were adjusted by gingivectomy and closed with a resorbable type surgical thread (3-0, Manufacture; Johnson and Johnson Intl. XHGHCGO) and simple interrupted sutures.

\section{Periodontal Surgical Procedure}

Intracrevicular incisions, full-thickness muco-periosteal flaps were raised vistibularly for defect access and granulation tissue removal on both sides of the teeth (Fig. 1A). For a better access or a better closure of the surgical sites, vertical releasing incisions (mesial and distal to the surgical site extended below the mucogingival junction) were performed. All granulation tissues were debrided from the root surfaces and the defects. One site was treated with torus mandibularis as autogenous bone graft and the other site was treated only with a full-thickness mucoperiosteal flap. The graft was delivered to the bony defect with a spatula and added in incremental fashion. Light pressure was used to maintain space between the graft particles to allow revascularization of the site. The defect was filled or slightly overfilled (Fig. 1C) to maximize regeneration without compromising flap closure or vascular supply. The flaps were closed and sutured with interrupted sutures (Fig. 1D). All patients received antibiotics for
1 week (Amoxicillin $500 \mathrm{mg} / 3$ times/day) and rinsed with $0.2 \%$ chlorhexidine solution twice daily. The sutures were removed 14 days postoperatively. All patients have been re-called after $3,6,9$, and 12 months for the clinical and radiographic assessment.

\section{Histochemical Analysis}

At the time of torus mandibularis removal, a small sample of the collected materials was fixed in $10 \%$ formalin, routinely processed and embedded in paraffin. Serial sections were cut at a thickness of $5 \mu \mathrm{m}$. Sections were stained with hematoxylin (HE) and Vankossa histochemical stain as described elsewhere. ${ }^{29}$

During the 8 month postoperative follow-up period, one case of the tested group involving teeth \#2, 3, 4 and 5 demonstrated severe pain in the un-restorable tooth \#2 due to deep carious lesion with pulp exposure. After consultation with endodontist it was decided to extract the tooth and approved by the ethics committee, College of Dentistry, University of Dammam. The research team surgically removed tooth \# 2 including its proximal alveolar bone (demarcated and removed by Pizotome) and was fixed in $10 \%$ formalin, decalcified in 5\% formic acid, routinely processed, sectioned at $5 \mu \mathrm{m}$ thickness and subjected to histochimical staining using HE and
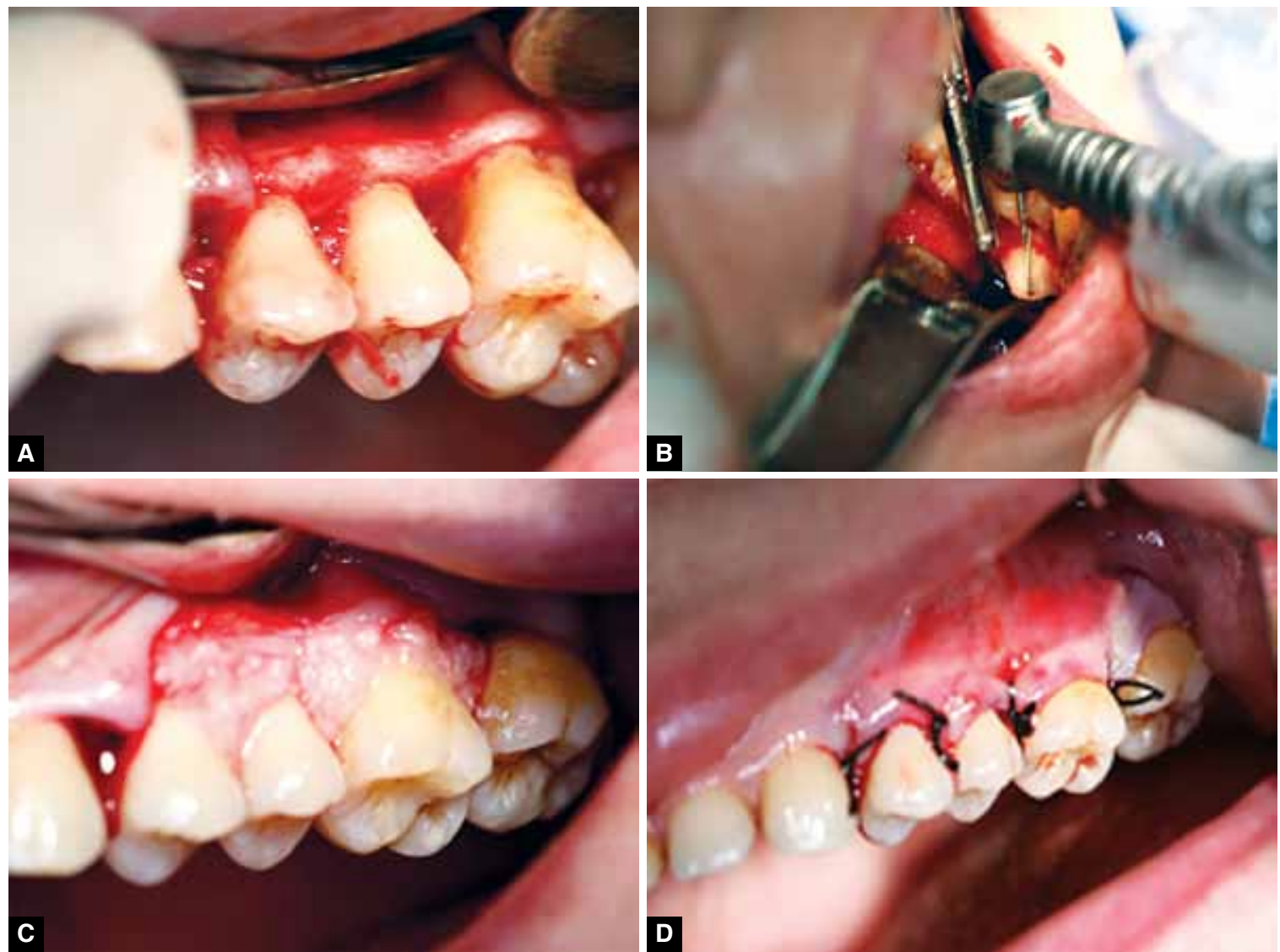

Figs 1A to D: (A) A sulcular incision full-thickness flap reflected, showing intrabony defects at the upper posterior teeth, (B) Torus mandibularis removed with surgical bur, and the bone grafts collected with the bone trap, (C) Torus mandibularis bone graft fills the intraossous defects and (D) Suturing the flap with interrupted sutures 
von Kossa that help to demonstrate the histopathologic background of our radiographic findings.

\section{Standardized Radiographs}

All the standardized radiographs were taken with the Rinn film holder. This device consists of film holder into which the dental radiograph fits, a plastic plate, which the patient bites on, and a rod connected to the film holder that protrudes from the patient's mouth to allow parallel alignment of the $X$-ray tube. ${ }^{30}$ To ensure that the patient's bite was reproducible in the standardized group of radiographs, an impression material (Henry Schein Blu-Bite Vinyl Polysiloxane Derivative Registration Material: Henry Schein Inc, Melville, NY, USA) was placed on the plastic bite block. The patient was instructed to bite on the bite block while holding his or her teeth in their posterior position (centric relation). Once the impression material set, the film holder was removed from the mouth. The film was then added; the patient set his or her teeth in the registered bite, and the X-ray was taken. Identical exposure parameters were used at all examinations, and the examination film was automatically processed. All periapical radiographs were digitized and saved in a tagged image file format (TIFF). Then, the bone density and marginal bone levels were measured by using Image J software. ${ }^{31}$

\section{Data Analysis}

Clinical data were expressed as means \pm and standard deviation (SD) for each variable and examined intervals. The change in clinical parameters from baseline to 3,6, 9 , and 12 months after surgery within each group were compared using the paired t-test. One-way analysis of variance was used to test the difference between groups. The level of significance for analysis was set at $\mathrm{p} \leq 0.05$.

Change in radiographic findings was done to evaluate increase in alveolar bone level (measured at significant level of $\mathrm{p}<0.001$ ) and bone density (measured at significant level of $\mathrm{p}<0.05$ ), parallel to the clinical evaluation, to estimate the progress in bone gain for each group. For clarification and optimization purposes, baseline radiographs for each case was digitally overlapped to 12-month postoperative one, using adobe Photoshop CS4 software, and the difference was measured as for bone density and height by image $\mathrm{j}$ software and the average was calculated and compared.

\section{RESULTS}

All patients completed the study without complications and recalled for evaluation at 3, 6, 9 and 12 months. The results of the present study showed no statistically significant differences in the clinical parameters measured at the baseline between the treated sites. With regard to the gingival index, statistical significant changes were observed after treatment with torus mandibularis (group I) comparing to the pretreatment period (baseline $2.1 \pm$ 0.21 , after 12 months $0,02 \pm 0.03$ ). In addition, there was a statistically significant change following treatment with torus mandibularis (group I) compared to sites treated with a full-thickness flap alone (group II) $(0,02 \pm 0.03$, $0.12 \pm 0.03$ respectively) (Table 1 ).

The present work illustrated a comparison between both groups with regard to probing depth and clinical attachment level. There was a gain in CAL of $88.4 \%$ (mean value at baseline of $5.12 \pm 0.52$, at 12 months $0.59 \pm 0.04$ $\mathrm{mm}$ ) for torus mandibularis sites, and $39.7 \%$ (mean value at baseline of $5.07 \pm 0.41$, at 12 months $3.06 \pm 0.06$ $\mathrm{mm}$ ) for full-thickness flap alone ( $\mathrm{p} \leq 0.01)$. With respect to probing depth, there was a reduction in probing depth of $75.4 \%$ (mean value at baseline of $7.62 \pm 0.42$, at 12 months $1.87 \pm 0.16 \mathrm{~mm}$ ) for torus mandibularis sites and $49.6 \%$ (mean value at baseline of $7.51 \pm 0.31$, at 12 months $3.78 \pm$ $0.31 \mathrm{~mm}$ ) for sites treated with a full-thickness flap alone ( $\mathrm{p} \leq$ 0.01) (Table 1 and Fig. 2).

A radiographic comparison of baseline and 12 months scores (Figs 3D and E) of the experimental group was

Table 1: Changes of the clinical parameters measurement in the test group (GI) and control group (GII)

\begin{tabular}{|c|c|c|c|c|c|c|c|c|c|}
\hline & \multirow{2}{*}{$\begin{array}{l}\text { Test group } \\
\text { GI (scores) }\end{array}$} & \multicolumn{2}{|c|}{ Control group } & \multirow{2}{*}{$\begin{array}{l}\text { Test group } \\
P D(\mathrm{~mm})\end{array}$} & \multicolumn{2}{|c|}{ Control group } & \multirow{2}{*}{$\begin{array}{l}\text { Test group } \\
A L(\mathrm{~mm})\end{array}$} & \multicolumn{2}{|c|}{ Control group } \\
\hline & & GI (scores) & $p$-value & & $P D(\mathrm{~mm})$ & $p$-value & & $A l(\mathrm{~mm})$ & $p$-value \\
\hline \multirow[t]{2}{*}{ Baseline } & 2.1 & 2.15 & NS & 7.62 & 7.51 & NS & 5.12 & 5.07 & NS \\
\hline & \pm 0.21 & \pm 0.23 & & \pm 0.42 & \pm 0.31 & & \pm 0.52 & \pm 0.41 & \\
\hline \multirow{2}{*}{$\begin{array}{l}\text { Three months } \\
\text { postoperative }\end{array}$} & 0.04 & 0.24 & * & 3.01 & 3.91 & * & 1.5 & 3.41 & * \\
\hline & \pm 0.03 & \pm 0.19 & & \pm 0.23 & \pm 0.44 & & \pm 0.13 & \pm 0.45 & \\
\hline \multirow{2}{*}{$\begin{array}{l}\text { Six months } \\
\text { postoperative }\end{array}$} & 0.06 & 0.23 & * & 2.76 & 3.82 & * & 1.32 & 3.09 & * \\
\hline & \pm 0.02 & \pm 0.21 & & \pm 0.21 & \pm 0.38 & & \pm 0.11 & \pm 0.36 & \\
\hline \multirow{2}{*}{$\begin{array}{l}\text { Nine months } \\
\text { postoperative }\end{array}$} & 0.03 & 0.21 & * & 2.12 & 3.8 & * & 1.11 & 3.11 & * \\
\hline & \pm 0.04 & \pm 0.19 & & \pm 0.18 & \pm 0.38 & & \pm 0.08 & \pm 0.37 & \\
\hline \multirow{2}{*}{$\begin{array}{l}\text { Twelve months } \\
\text { postoperative }\end{array}$} & 0.02 & 0.12 & * & 1.87 & 3.78 & * & 0.59 & 3.06 & * \\
\hline & \pm 0.03 & \pm 0.16 & & \pm 0.16 & \pm 0.31 & & \pm 0.04 & \pm 0.33 & \\
\hline
\end{tabular}

NS: Nonsignificant differences; *: Significant differences 


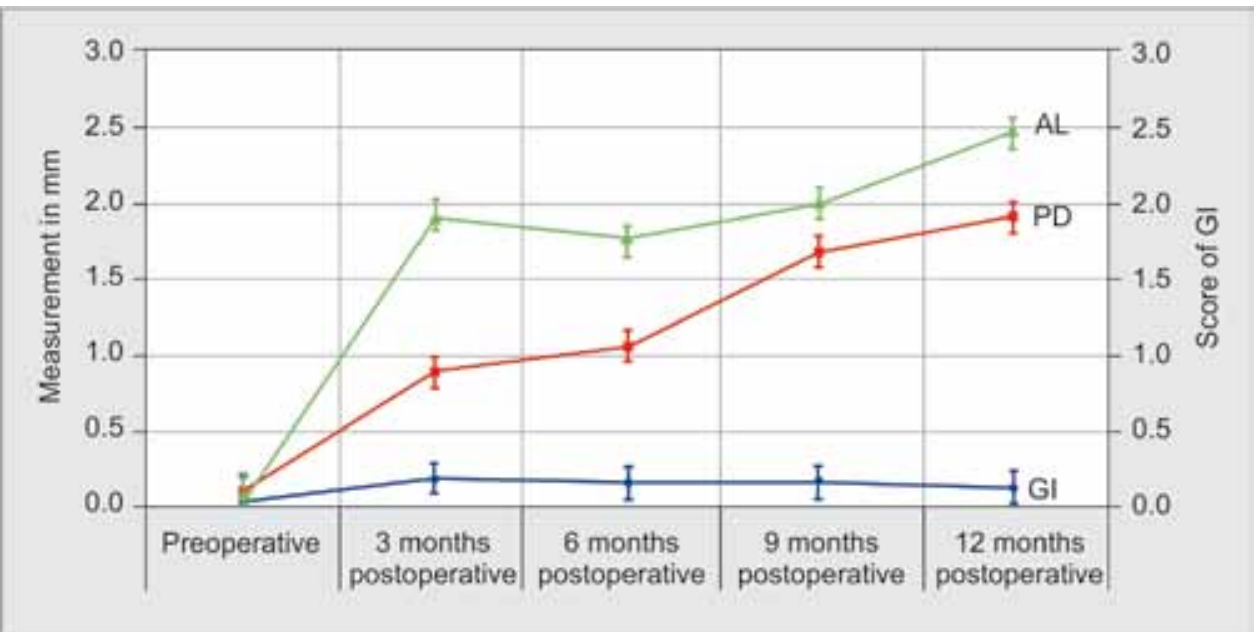

Fig. 2: The clinical parameters changes following treatment with mandibular tori (group I) comparing to a full-thickness flap alone (group II)

done by overlapping the two images (Fig. 3F). Similar procedure was done for the control group (Figs 3A to C). Our results show increase in alveolar bone level and density in experimental group (Fig. 3F) compared to the control group (Fig. 3C). Differences between and the test and control groups for bone density was (17\%) as expressed in percentage of difference in pixel $/ \mathrm{mm}^{2}$ (Fig. 4) that was significant at $\mathrm{p}<0.05$.

Histopathologically, HE staining supports our hypothesis that the torus mandibularis contains active osteoblasts that were mainly located at the surface of preoperative sample as shown in (Fig. 5A). Higher magnification (Figs 5B and C) demonstrates the active osteoblasts with active production of bone matrix that stain deep violet in HE stain when compared to the main eosinophilic structure of lamellar bone of torus.

The proximal alveolar bone of the extracted tooth \#2 at 9th months postoperatively demonstrate clumped osteoblasts surrounding the eosinophilic zone on $\mathrm{HE}$ staining (Fig. 6A) interrupted by violet materials presenting active osteogenesis that focally stain black with van Kossa shown in (Fig. 6B) representing mineralization process of the newly formed bone.

\section{DISCUSSION}

The ambitious goal of periodontal therapy is to restore the periodontal attachment apparatus to its prediseased state. The proper placement of graft materials for the infrabony defects are one mode of therapy that attempts to restore the lost periodontal attachment apparatus. The bone graft material provides regeneration through inductive or conductive processes. The conductive graft act as scaffold to support the new tissue growth and replaced by the host tissue. The inductive graft stimulates the host tissues to regenerate the lost structures.
All medical devices that come into a direct contact with living tissues must be composed of biologically compatible materials. They must not cause either systemic or local toxicity and should not be carcinogenic or genotoxic. In addition, they must not affect the blood clotting mechanisms and should not induce immune responses.

The present study was designed to evaluate the clinical significance of using mandibular tori as a graft material in the treatment of intraosseous defects in patients with chronic periodontitis.

The present study focused on deep intrabony defects characterized by clinical attachment level and probing pocket depth not less than $6 \mathrm{~mm}$. This is in agreement with the other investigations, which demonstrated a positive interrelationship between defect depth, clinical attachment level gain and osseous fill after the indicated periodontal therapy. ${ }^{32-34}$ In addition, each patient included in the present study served as their own control by the use of split mouth techniques to avoid the individual variations as a vulnerable point in such studies. Moreover, this method is limited to patients only having torus mandibularis.

It is important to note that, several factors might dictate important effects on the outcome of the regenerative therapy. These factors are: age, patient's gender, oral hygiene, compliance to postoperative instructions and care, surgical manipulation and perfect, defect characteristics and severity. ${ }^{6}$ However, in a split-mouth design these factors have a similar influence on both therapeutic modalities to be compared. ${ }^{35}$

A sterile and disposable aspiration set with a plastic suction tube and filter equipped with an internal mesh with a pore diameter of $300 \mu \mathrm{m}$ was used to collect bone fragments in this study. The results of the present study recorded that there were no problems with filter blockage during collection. This is in agreement with a study 

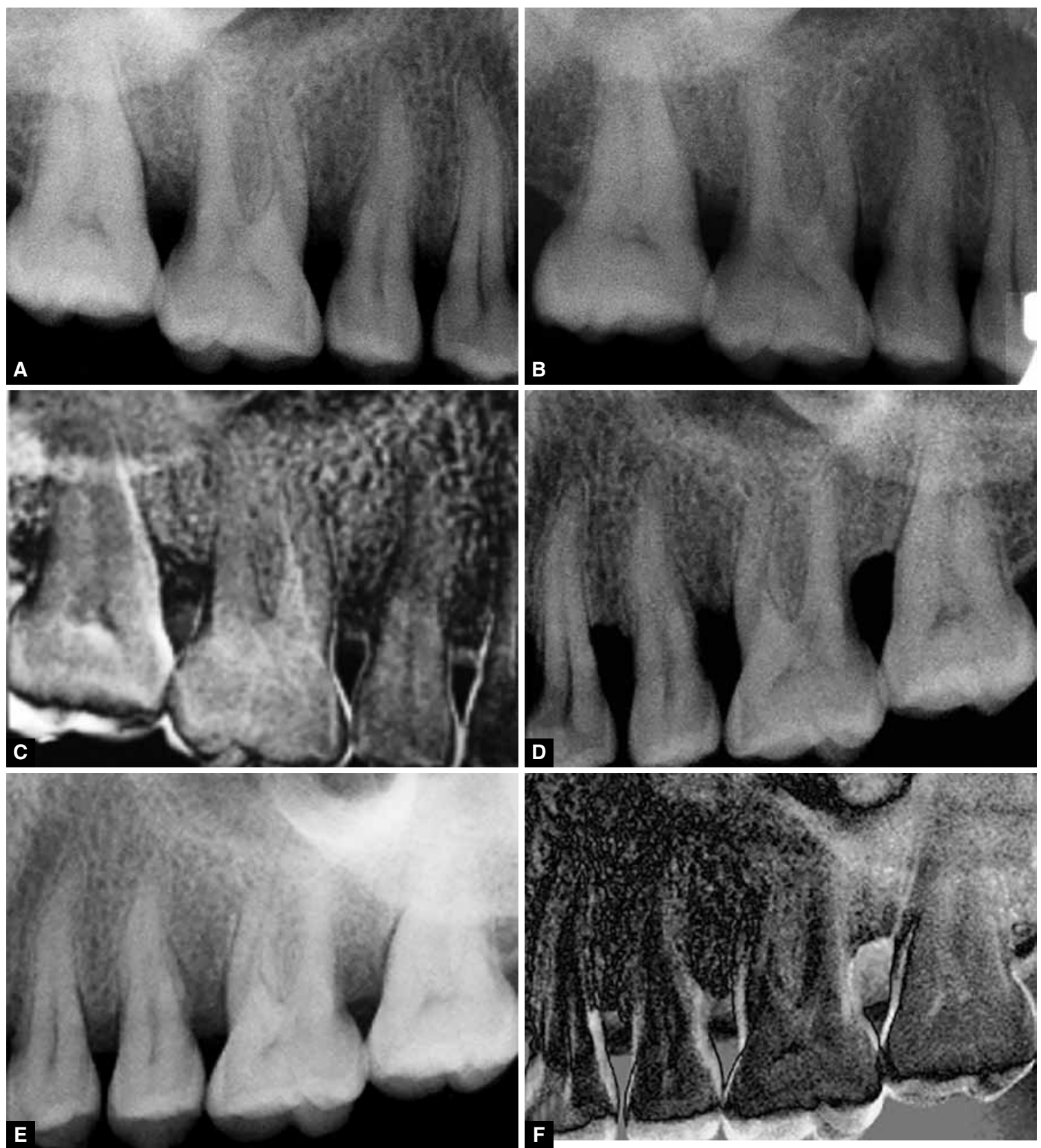

Figs 3A to F: Radiographic follows up for a full-thickness flap alone shown in (A, B and C): (A) Represents the preoperative radiograph, (B) Shows 12 months postoperative progress in the same field, (C) Demonstrates the difference in bone gain within the 12 months follow-up by overlapping $A$ and $B,(D, E$ and $F$ ) showing radiographic follow-up for torus mandibularis, (D) represents the preoperative radiograph, $(E)$ shows 12 months postoperative progress in the same field, $(F)$ demonstrates the difference in bone gain within the 12 months follow-up by overlapping $\mathrm{D}$ and $\mathrm{E}$. The progress in bone gain in regards of bone density and height clearly demonstrate a significant difference of torus mandibularis bone graft compared to a full-thickness flap alone

performed by Sivolella et $\mathrm{al}^{28}$ who demonstrated that $300 \mu \mathrm{m}$ meshes are capable of catching most of the suctioned material, without the problems of fragment blocking of the aspiration system from obstruction of the filter. In addition, by the use of a two distinct aspiration tubes for bone chip collection and control of salivation and bleeding, the bacterial contamination can be reduced. A study by Etchenson et a $1^{35}$ demonstrated that osseous coagulum collected in bone traps had potential for bacterial contamination. They also suggested methods for decontamination. Their results indicate significant bacterial contamination of osseous coagulum. They have shown that bone traps used with stringent surgical protocols that include segregated suction tips and pre-procedural chlorhexidine rinsing, with tetracycline will have a significant, however, incomplete influence on the reduction of bacterial contamination of osseous coagulum. The same protocol as the one used by Etchenson et $\mathrm{al}^{35}$ was used in this study. The initial therapy period was 4 weeks. In the Etchenson study, the initial treatment phase was not utilized for procuring graft material. Moreover, Tezulas et $\mathrm{al}^{36}$ demonstrated the decontamination of autogenous bone grafts collected during dental implant site preparation by using clindamycin or 


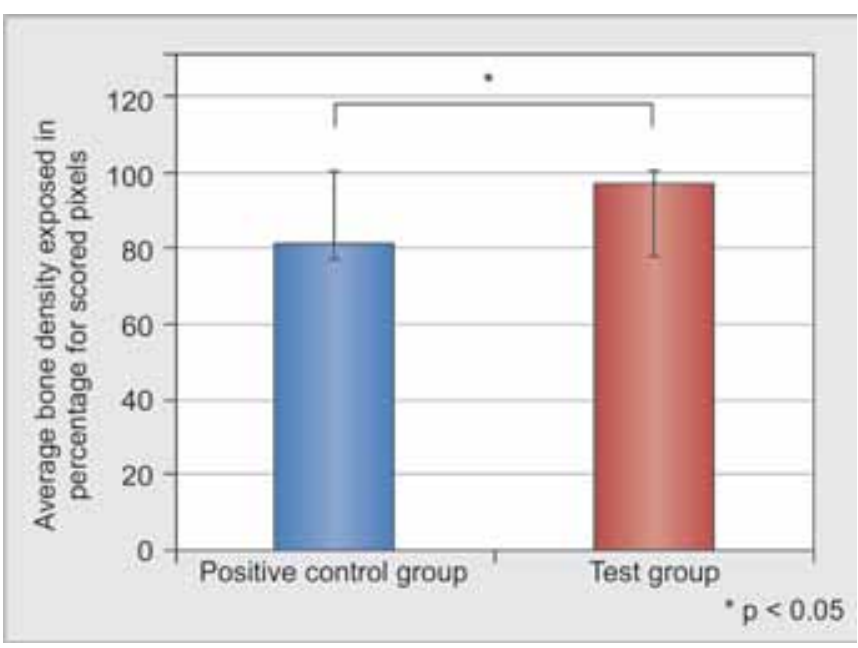

Fig. 4: Diagram show, the bone density (in pixel/ $/ \mathrm{mm}^{2}$ ) following treatment with mandibular tori (group I) comparing to a fullthickness flap alone (group II)

chlorhexidine solutions. Their results showed that both agents effectively decontaminated the collected bone particles. In this study, the protocol was utilized two surgical suction tips: one to aspirate saliva and blood and another to collect osseous coagulum from the osteotomy site.

The gingival index remained reduced when compared to the baseline, throughout the study for both groups, with a statistically significant difference between the groups.

It was suggested that the clinical objectives of periodontal regeneration include: increased bone height, gain in clinical attachment levels, probing depth reduction, improved esthetics and long-term maintenance of teeth in health and comfort. ${ }^{37,38}$ In the present study, a significant reduction in pocket probing depth and clinical attachment gain was obtained when using of mandibular tori as autogenous bone graft. These results are in accordance with the study performed by Scott and Ganz ${ }^{39}$ which claimed that mandibular tori could be used as the donor sites of autogenous grafting materials to fill the remaining defects around implants installed in extraction wounds. In addition, the results of the present study showed a significant reduction in pocket depth and attachment level gain when using mandibular tori compared to a full-thickness flap alone.

Contrary to the accepted concept, our results show that mandibular tori can be used successfully in the treatment of advanced periodontal diseases. Histochemical analysis of torus bone indicates the presence of active osteoblasts mainly on the bone surface. A study by Scott

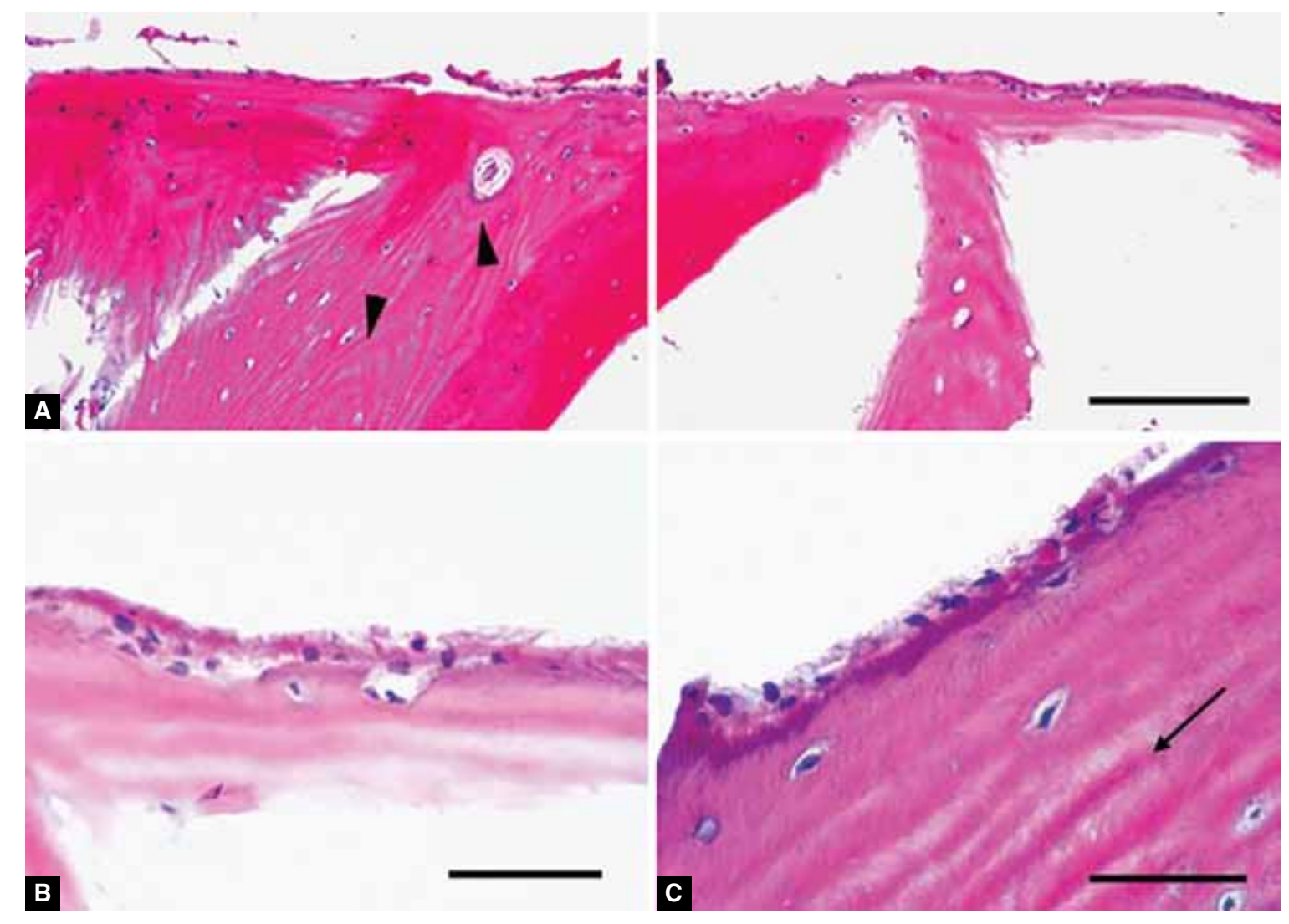

Figs 5A to C: Histopathology of torus mandibularis, (A to C) Hematoxylin and eosin (HE), Bars indicate $300 \mu \mathrm{m}$ (A), and $50 \mu \mathrm{m}$ (B and $\mathrm{C})$, respectively. Torus mandibularis is made-up of lamellar type of bone. Torus mandibularis histologically consist of central haversian system of concentric layers around central nutrient canals and flattened layers toward the surface similar to cortical plate of bone (A). The peripheral layers of bone are covered with osteoblasts actively synthesizing osteoid matrix as indicated by the deep violet staining, which are newly formed or are in the process of formation (B and $C)$. Arrow head indicate concentric layers around central canal. Tailed arrow head indicate the flattened layer of bone trabeculae toward surface 


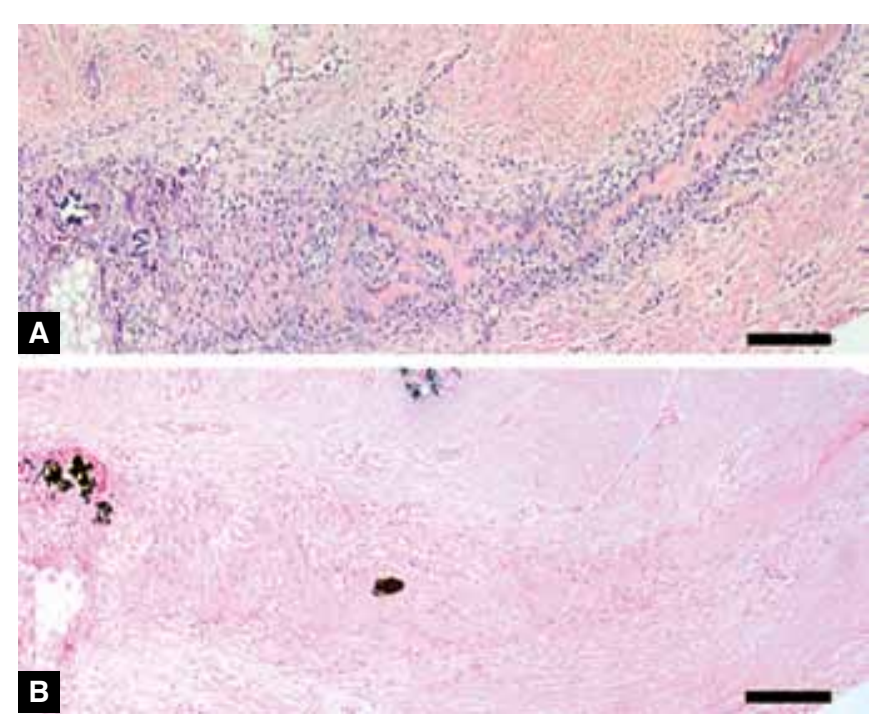

Figs 6A and B: Histopathology: (A) Hematoxylin and Eosin (HE) and $(B)$ von Kossa stain show clumped osteoblasts with active bone deposition as black globules stained by von Kossa that illustrate the ability of torus-derived osteoblasts to survive, differentiate and function in the grafted sites

and $\mathrm{Ganz}^{39}$ showed osteoinduction through the use of active osteoblasts around dental implants. To the best of our knowledge, this is the first report on using active osteoblasts of torus mandibularis to induce bone formation for treatment of periodontal bony defects. The function of tori-derived cells and their precursors are the only possible explanation why group I, where the torus mandi-bularis bone graft was implanted in the bony defect, show better results. On the contrary Kainulainen et $\mathrm{al}^{40}$ reported that considerable amounts of bone can be harvested from tori with a suction trap. If a torus is removed as a block it can be particulates with a bone mill or used as a block graft. Therefore, mandibular tori are composed of very dense cortical bone and are not ideal for use as block onlay or inlay grafts. The tori are difficult to shape, mortise and fixate to the host bone.

Lines from previous studies documented the inhibitory effect of periodontal disease-induced inflammatory mediators on differentiation and function of existing osteoblasts. Our results show that just elimination of the inflammatory mediators in the control sides is not enough for bone to regain its vitality and osteogenic capabilities. Such observation is the only possible explanation for the difficulty in bone gain of advanced periodontal diseases treated with full-thickness flap. On the contrary, introducing torus bone, rich with osteoblasts and its precursors, results in tremendous bone gain and decrease pocket depth that was histopathologically proven to be due to differentiation and activation of introduced osteoblasts.

In general, successful periodontal therapy means regeneration and/or maintenance of periodontal support. Complete pocket elimination by regeneration or pocket resolution is impossible. The results of the present study showed that it was possible to reduce the probing pocket depth in sites treated with mandibular tori, thus allowing more effective maintenance therapy. The present study suggested that mandibular tori autogenous bone graft could provide benefits as a periodontal therapeutic modality. Furthermore, the radiographic bone gains recorded after treatment with mandibular tori confirmed the clinical observations. The present study show that the use of mandibular tori as bone grafting material may provide additional benefits in the treatment of advanced periodontal disease; and enhance the regenerative potential of periodontal intraosseous defects.

\section{ACKNOWLEDGMENT}

The authors would like to express many thanks for assistant staff in College of Dentistry, and all staff members of general pathology, College of Medicine, University of Dammam, Dammam, Saudi Arabia.

\section{REFERENCES}

1. Weltman R, Trejo PM, Morrison E, Caffesse R. Assessment of guided tissue regeneration procedures in infrabony defects with bioabsorbable and non-resorbable barriers. J Periodont 1997;68(6):582-588.

2. Falk H, Laurell L, Ravald N, Teiwik A. Persson guided tissue regeneration therapy of 203 consecutively treated intrabony defects using a bioabsorbable matrix barrier. Clinical and radiographic findings. J Periodont 1997;68(6):571-578.

3. Lovelace TB, Melloning JT, Meffert RM, Jones AA, Nummikoski PV, Cochran DL. Clinical evaluation of bioactive glass in the treatment of periodontal osseous defects in humans. J Periodont 1998;69(9):1027-1033.

4. Eickholz P, Kim TS, Steinbrenner H, Dorfer C, Holle R. Guided tissue regeneration with bioabsorbable barriers: Intrabony defects and class II furcations. J Periodont 2000;71(6):999-1008.

5. Garrett S, Bogle G. Periodontal regeneration. A review of flap management. Periodont 20001993 Feb;1:100-108.

6. Karring T, Lindhe J, Cortellini P. Regenerative periodontal therapy. In: Lindhe J, Karring T, Lang NP, editors. Clinical Periodontology and Implant Dentistry. 3rd ed. Copenhagen: Munksgaard, 1998. p. 597-646.

7. Brunsvold MA, Mellonig JT. Bone grafts and periodontal regeration. Periodont 2000 1993;1(1):80-91.

8. Yukna RA. Synthetic bone grafts in periodontics. Periodont 2000 1993;1(1):92-99.

9. Dragoo MR, Sullivan HC. A clinical and histological evaluation of autogenous iliac bone grafts in humans: Part I. Wound healing 2 to 8 months. J Periodont 1973;44(10):599-613.

10. Hiatt $W H$, Schallhorn RG, Aaronian AJ. The induction of new bone and cementum formation. IV. Microscopic examination of the periodontium following human bone and marrow allograft, autograft and nongraft periodontal regenerative procedures. J Periodont 1978;49(10):495-512.

11. Stahl SS, Froum SJ, Kushner J. Healing responses of human intraosseous lesions following the use of debridement, grafting and citric acid root treatment. II. Clinical and histologic observations one year post surgery. J Periodont 1983;54(6): 325-328. 
12. Bowers GM, Chadroff B, Carnevale R, et al. Histologic evaluation of new attachment apparatus in humans. Part II. J Periodont 1989;60(12):676-682.

13. Bowers GM, Chadroff B, Carnevale R, et al. Histologic evaluation of new attachment apparatus in humans. Part III. J Periodont 1989;60(12):683-693.

14. Coretellini P, Tonetti MS. Focus on intrabony defects: guided tissue regeneration. J Periodont 2000;22(1):104-132.

15. Ellegaard B, Low H. New attachment of periodontal tissues after treatment of intrabony lesions. J Periodont 1971; 42(10):648-652.

16. Froum SJ, Kushner L, Stahl SS. Healing responses of human intraosseous lesions following the use of debridement, grafting and citric acid root treatment. I. Clinical and histologic observations six months postsurgery. J Periodont 1983;54(2):67-76.

17. Hawley CE, Miller J. A histological examination of a free osseous autograft: case report. J Periodont 1975;46(5):289-293.

18. Nabers CL, O'Leary TJ. Autogenous bone grafts: case report. Periodontics 1967;5(4):251-253.

19. Hiatt WH, Schallhorn RG. Intraoral transplants of cancellous bone and marrow in periodontal lesions. J Periodont 1973;44(4):194-208.

20. Rosenberg MM. Free osseous tissue autografts as a predictable procedure. J Periodont 1971;42(4): 195-209.

21. Neville BW, Damm DD, Allen CM, Bouqout JE, editors. Oral and maxillofacial pathology. Philadelphia: WB Saunders Co 1995. p. 17-20.

22. Demetries Z, Maria B, Panayiotis P. Concurrence of torus palatines with palatal and buccal exostoses: case report and review of the literure. Oral Surg Oral Med Oral Pathol Oral Radiol Endod 1998;85(5):552-557.

23. Sapp JP, Eversole LR, Wysocki GP, editors. Contemporary oral and maxillofacial pathology. St. Louis: Mosby 1997. p. 106-107.

24. Regezi JA, Sciubba JJ. Oral pathology: clinical-pathologic correlations. Philadelphia: WB Saunders Co 1989. p. 386-387.

25. Furst GM. Toms mandibularis bei den Eskimos und anderen Rassen. Anat Anz 1908;32:295-306.

26. Loe H, Sillness J. Periodontal disease in pregnancy. Acta Odontologica Scandinavica 1963 Dec 21;533.

27. Ramfjord SP. The periodontal disease index (PDI). J Periodont 1967;38(6):602-610.

28. Sivolella S, Berengo M, Scarin M, Mella F, Martinelli F. Autogenous particulate bone collected with a piezoelectric surgical device and bone trap: microbiological and histomorphometric study. Archives of Oral Biology 2006;51 (10):881-883.
29. Bonewald LF, Harris SE, Rosser J, Dallas MR, Bosky A. Von Kossa staining alone is not sufficient to confirm that mineralization in vitro represents bone formation. Calcified Tissue International 2003;72(5):537-547.

30. McDonald SP. A method to reduce interproximal overlapping and improve reproducibility of bitewing radiographs for use in clinical trials. Community Dent Oral Epidemol 1983;11 (5):289-295.

31. Burger B. Digital image processing —an algorithmic approach using Java. Springer-Verlag New York. ISBN 2008;978-1-84628379-6.

32. Cortellini P, Bowers GM. Periodontal regeneration of intrabony defects: an evidence-based treatment approach. Int J Periodont and Rest Dent 1995;15(2):128-145.

33. Laurell L, Gottlow J, Zybutz M, Perrson R. Treatment of intrabony defects by different surgical procedures: a literature review. J Periodont 1998;69(3):303-310.

34. Eickholz P, Kim TS, Holle R, Hausmann F. Long-term results of guided tissue regeneration therapy with non-resorbable and bioabsorbable barriers. Class II furcations. J Periodont 2001;72(1):35-42.

35. Etcheson AW, Miley MD, Gillespie MJ. Osseous coagulum collected in bone traps: potential for bacterial contamination and methods for decontamination. J Oral Implantol 2007; 33(3):109-115.

36. TezulasE, Dilek OC, Topcuoglu N,KulekciG. Decontamination of autogenous bone grafts collected during dental implant site preparation: a pilot study. Oral Surg Oral Med Oral Pathol Oral Radiol Endod 2009;107(5):656-660.

37. Nygaard-Østby P, Bakke V, Nesdal O, Susin C, MEWikesjö U. Periodontal healing following reconstructive surgery: effect of guided tissue regeneration using a bioresorbable barrier device when combined with autogenous bone grafting. A randomized controlled trial 10-year follow-up. J Clin Periodontol 2010;37(4):366-373.

38. Selcuc Y, Cakar G, Yildirim B, Sculean A. Healing of two and three wall intrabony periodontal defects following treatment with an enamel matrix derivative combined with autogenous bone. J Clin Periodontol 2010;37(6):544-550.

39. Scott D, Ganz DMD. Mandibular tori as a source for onlay bone graft augmentation: a surgical procedure. The Implant Report 1997;9(9):973-984.

40. Kainulainen VT, Sàndor GKB, Oikarinen KS, Clokie CML. The intraoral bone harvesting sites for osseous reconstruction in oral and maxillofacial surgery. Oral Health 2003;93(5): 10-24. 